\title{
Entropy methods for reaction-diffusion equations: slowly growing a-priori bounds
}

\section{Laurent Desvillettes and Klemens Fellner}

\begin{abstract}
In the continuation of [8], we study reversible reaction-diffusion equations via entropy methods (based on the free energy functional) for a 1D system of four species. We improve the existing theory by getting 1) almost exponential convergence in $L^{1}$ to the steady state via a precise entropy-entropy dissipation estimate, 2 ) an explicit global $L^{\infty}$ bound via interpolation of a polynomially growing $H^{1}$ bound with the almost exponential $L^{1}$ convergence, and 3), finally, explicit exponential convergence to the steady state in all Sobolev norms.
\end{abstract}

\section{Introduction}

\subsection{General presentation}

This paper is part of a general study of the large-time behaviour of diffusive and reversible chemical reactions of the type

$$
\alpha_{1} \mathcal{A}_{1}+\cdots+\alpha_{q} \mathcal{A}_{q} \rightleftharpoons \beta_{1} \mathcal{A}_{1}+\cdots+\beta_{q} \mathcal{A}_{q} \quad \alpha_{i}, \beta_{i} \in \mathbb{N}
$$

in a bounded box $\Omega \subset \mathbb{R}^{N}(N \geq 1)$.

Systems of type (1.1) are well known in the numerous literature on reaction-diffusion systems. For the large time behaviour of global classical solutions (e.g. within invariant domains) we refer, for instance, to [31, 4] and the references therein. For global weak solutions see e.g. [24, 28, 29] with references. Many authors (e.g. [37, 23, 21, 36, 20, 22, 27] and the references

2000 Mathematics Subject Classification: 35B40, 35K57.

Keywords: Reaction-diffusion, entropy method, exponential decay, slowly growing a-priori estimates. 
therein) have deduced compactness and a-priori bounds from Lyapunov functionals. We recall in particular $[25,15,16]$, where generalized Lyapunov structures of reaction-diffusion systems yield a-priori estimates to establish global existence of solutions. We mention also [30, 26] and the references therein where peculiar Lyapunov functionals are designed to show optimized stability and instability properties for reaction-diffusion systems.

As in [8], we exploit as much as possible the free energy functional of these systems. The basic idea consists in studying the large-time asymptotics of a dissipative PDE by looking for a nonnegative Lyapunov functional $E(f)$ and its nonnegative dissipation $D(f)=-\frac{d}{d t} E(f(t))$ along the flow of the PDE, which are well-behaved in the following sense: firstly, $E(f)=0 \Longleftrightarrow f=f_{\infty}$ for some equilibrium $f_{\infty}$ (usually, such a result is true only when all the conserved quantities have been taken into account), and secondly, there exists an entropy/entropy-dissipation estimate of the form $D(f) \geq \Phi(E(f))$ for some nonnegative function $\Phi$ such that $\Phi(x)=0$ $\Longleftrightarrow x=0$. If $\Phi^{\prime}(0) \neq 0$, one usually gets exponential convergence toward $f_{\infty}$ with a rate which can be explicitly estimated.

This line of ideas, sometimes called the "entropy method", is an alternative to the linearization around the equilibrium and has the advantage of being quite robust. This is due to the fact that it mainly relies on functional inequalities which have no direct link with the original PDE.

The entropy method has lately been used in many situations: nonlinear diffusion equations (such as fast diffusions [7, 6], equations of fourth order [2], Landau equation [11], etc.), integral equations (such as the spatially homogeneous Boltzmann equation [33, 34, 35]), or kinetic equations ([3], $[12,13],[14])$.

In the context of reaction-diffusion systems, especially in the theory of semiconductors, the entropy method has been used e.g. in [19, 17, 18]. In [17], for instance, exponential convergence towards equilibrium for general systems of the type (1.1) (coupled with an equation for the electric potential) was shown provided that globally existing solutions are known. Note that in general global existence of weak solutions for systems of type (1.1) is unknown and that boundedness of the entropy is insufficient to guarantee that the reaction terms belong to $L^{1}$ (see [9]).

At variance with the work that we propose here, the method of proof in [17] is based on a contradiction argument which does not lead to explicit constants. With the above notation, it is shown there that assuming a sequence of functions $f_{n}$ such that $D\left(f_{n}\right)=C_{n} E\left(f_{n}\right)$ for constants $C_{n} \rightarrow 0$ as $n \rightarrow \infty$, and such that $E\left(f_{n}\right)$ is bounded, it is possible to extract a subsequence of $f_{n}$ which converges to a limit, causing finally a contradiction (once the conservation laws have been taken into account). 
In our previous paper [8], we have proven quantitative exponential convergence to equilibrium with explicit rates (all constants are also explicit) for the systems modelling the reactions $2 \mathcal{A}_{1} \rightleftharpoons \mathcal{A}_{2}$ and $\mathcal{A}_{1}+\mathcal{A}_{2} \rightleftharpoons \mathcal{A}_{3}$. The proven entropy/entropy-dissipation estimate used global $L^{\infty}$ bounds on the concentrations, which are known for these systems (they are consequences of maximum principle type properties).

In this paper, we prove exponential convergence in $L^{1}$ (and consequently for any Sobolev norms) for a system with four species

$$
\mathcal{A}_{1}+\mathcal{A}_{3} \rightleftharpoons \mathcal{A}_{2}+\mathcal{A}_{4},
$$

for which a global $L^{\infty}$ bound was so far - up to our knowledge - unknown, but for which, at least in $1 \mathrm{D}$, a polynomially growing $L^{\infty}$ bound can be established. We focus on this particular system to present in a simple way the proposed method, which is our primary aim rather than the actual asymptotic result.

Note that for the equation that we consider, existence and uniqueness of classical solutions in 1D is a consequence of [1] and [25, theorem 2.4]. Global existence of weak solutions in any dimension follows e.g. from [28].

The method that we present here is very different from the tools used in these works and can be summarized in this way: Firstly, we prove a polynomially growing $L^{\infty}$ bound for the solution of our equation (this a priori bound is therefore called "slowly growing"). Then, we establish a precise entropy/entropy-dissipation estimate, for which the constant depends logarithmically on the $L^{\infty}$ norm of the solution thanks to a somewhat lengthy, but elementary computation. Thus, a Gronwall type lemma implies "almost exponential" decay in $L^{1}$ towards the steady state. Secondly, we prove an explicit, uniform in time $L^{\infty}$ bound by interpolation of the almost exponential $L^{1}$ decay with a polynomially growing $H^{1}$ bound. Finally, thanks to this global $L^{\infty}$ bound, the entropy/entropy-dissipation estimate can be used a second time and yields exponential decay towards the steady state.

Note that slowly growing a priori bounds have already been used in the context of entropy methods in kinetic theory (cf. [34]), as well as interpolation between an explicit decay in weak norm and controlled growth in strong norm (cf. [10]). The last step (getting the exponential decay) is however a new result in the context of entropy methods.

To state the problem, we denote with $a_{i} \equiv a_{i}(t, x) \geq 0, i=1,2,3,4$, the concentrations of the species $\mathcal{A}_{i}$ at time $t \geq 0$ and point $x \in \Omega$ ( $\Omega$ is a bounded interval of $\mathbb{R}$ ), and assume that reactions (1.2) are taken into account according to the principle of mass action kinetics, which leads to the system

$$
\partial_{t} a_{i}-d_{i} \partial_{x x} a_{i}=(-1)^{i}\left(l a_{1} a_{3}-k a_{2} a_{4}\right),
$$


with the strictly positive reaction rates $l, k>0$ and with $a_{i}$ satisfying homogeneous Neumann conditions

$$
\forall x \in \partial \Omega, t \geq 0, \quad \partial_{x} a_{i}(t, x)=0,
$$

and the nonnegative initial condition

$$
\forall x \in \Omega, \quad a_{i}(0, x)=a_{i, 0}(x) \geq 0 .
$$

Without loss of generality - we assume

$$
l=k=1, \quad|\Omega|=1,
$$

thanks to the rescaling $t \rightarrow \frac{1}{k} t, x \rightarrow|\Omega| x, a_{i} \rightarrow \frac{k}{l} a_{i}$. Finally, thanks to a translation, we can suppose that $\Omega=[0,1]$.

The solutions of (1.3)-(1.5) conserve the masses, that we assume to be strictly positive:

$$
M_{j k}=\int_{\Omega}\left(a_{j}(t, x)+a_{k}(t, x)\right) d x=\int_{\Omega}\left(a_{j, 0}(x)+a_{k, 0}(x)\right) d x>0,
$$

where we introduce the indices $j \in\{1,3\}$ and $k \in\{2,4\}$. Note that only three of the four $M_{j k}$ 's can be chosen independently since they are linked via the total mass

$$
M=M_{12}+M_{34}=M_{14}+M_{32} .
$$

Moreover, the conserved quantities provide naturally the following bounds

$$
\begin{aligned}
& \sup _{t \geq 0} \int_{\Omega} a_{j}(t, x) d x \leq \min _{k \in\{2,4\}}\left\{M_{j k}, M_{j k}\right\}:=M_{j}, \\
& \sup _{t \geq 0} \int_{\Omega} a_{k}(t, x) d x \leq \min _{j \in\{1,3\}}\left\{M_{j k}, M_{j k}\right\}:=M_{k} .
\end{aligned}
$$

When all the diffusivity constants $d_{i}$ are strictly positive, there exists a unique equilibrium state $a_{i, \infty}$ for (1.3) - (1.6) satisfying (1.7). It is defined by the unique positive constants solving $a_{1, \infty} a_{3, \infty}=a_{2, \infty} a_{4, \infty}$ provided $a_{j, \infty}+$ $a_{k, \infty}=M_{j k}$ for $(j, k) \in(\{1,3\},\{2,4\})$, that is:

$$
\begin{array}{ll}
a_{1, \infty}=\frac{M_{12} M_{14}}{M}>0, & a_{3, \infty}=M_{32}-\frac{M_{12} M_{32}}{M}=\frac{M_{32} M_{34}}{M}>0, \\
a_{2, \infty}=\frac{M_{12} M_{32}}{M}>0, & a_{4, \infty}=M_{14}-\frac{M_{12} M_{14}}{M}=\frac{M_{14} M_{34}}{M}>0 .
\end{array}
$$


Finally, we introduce the entropy (free energy) functional $E\left(a_{i}\right)$ and the entropy dissipation $D\left(a_{i}\right)=-\frac{d}{d t} E\left(a_{i}\right)$ associated to (1.3) - (1.6):

$$
\begin{aligned}
& E\left(a_{i}\right)=\int_{\Omega} \sum_{i=1}^{4} a_{i}\left(\ln \left(a_{i}\right)-1\right) d x \\
& D\left(a_{i}\right)=4 \sum_{i=1}^{4} d_{i} \int_{0}^{T} \int_{\Omega}\left|\partial_{x} \sqrt{a_{i}}\right|^{2} d x d t+\int_{\Omega}\left(a_{1} a_{3}-a_{2} a_{4}\right) \ln \left(\frac{a_{1} a_{3}}{a_{2} a_{4}}\right) d x .
\end{aligned}
$$

Outline of the paper: In section 2, we start by studying a priori bounds entailed by the decay of the entropy functional. These bounds allow to bootstrap an explicit, polynomially-growing (in time) $L^{\infty}$ bound on the concentrations $a_{i}$ (proposition 2.1), implying global existence of classical solutions (this result of existence can be proven by other means, cf. for example $[1,25])$.

In section 3, we establish an entropy/entropy-dissipation estimate with a constant depending logarithmically on the (polynomially growing) $L^{\infty}$ bound (proposition 3.1).

Hence, by a Gronwall lemma, we obtain in section 4 (proposition 4.2) an almost exponential decay in $L^{1}$ towards the steady state $a_{i, \infty}$ of the form

$$
\sum_{i=1}^{4} M_{i}^{-1}\left\|a_{i}(t, \cdot)-a_{i, \infty}\right\|_{L^{1}([0,1])}^{2} \leq 2 \sqrt{2}\left(E\left(a_{i, 0}\right)-E\left(a_{i, \infty}\right)\right) e^{-\frac{C_{1} t}{\ln (e+t)}}
$$

with a constant $C_{1}$ which can be computed explicitly (cf. appendix 5 ).

Furthermore, the almost exponential decay interpolates with a polynomially growing $H^{1}$ bound and we obtain an explicit, uniform in time $L^{\infty}$ bound (1.13). Finally, in return, exponential decay towards the steady state can be proven, and we obtain our main theorem:

Theorem 1.1 Let $\Omega$ be the interval $[0,1]$, and let $d_{i}>0$ for $i=1,2,3,4$ be strictly positive diffusion rates. Let the initial data $a_{i, 0}$ be nonnegative functions of $L^{\infty}(\Omega)$ with strictly positive masses $M_{j k}$ (defined by (1.7)) for $(j, k) \in(\{1,3\},\{2,4\})$. Then, the unique classical solution $a_{i}$ of $(1.3)-(1.6)$ is globally bounded in $L^{\infty}$ :

$$
\left\|a_{i}(t)\right\|_{L^{\infty}(\Omega)} \leq C_{2, i}
$$

and decay exponentially towards the steady state $a_{i, \infty}$ given in (1.9)-(1.10):

$$
\sum_{i=1}^{4} M_{i}^{-1}\left\|a_{i}(t, \cdot)-a_{i, \infty}\right\|_{L^{1}(\Omega)}^{2} \leq 2 \sqrt{2}\left(E\left(a_{i, 0}\right)-E\left(a_{i, \infty}\right)\right) e^{-C_{3} t},
$$

where $C_{2, i}$ and $C_{3}$ can be computed explicitly (Cf. appendix 5). 
Remark 1.1 Note that exponential decay towards equilibrium in all Sobolev norms follows subsequently by interpolation of the decay of Theorem 1.1 with polynomially growing $H^{k}$ bounds, which follow iteratively for $k>1$ from (1.13) and (4.5) inserted into the Fourier-representation used in lemma 2.3 and presented in appendix 5 (Sobolev norms of any order are created even if they do not initially exist, thanks to the smoothing properties of the heat kernel).

Notations: The letters $C, C_{1}, C_{2, i}, \ldots$ denote various positive constants (most of them are made explicit in appendix 5). It will also be convenient to introduce capital letters as a short notation for square roots of lower case concentrations and overlines for spatial averaging (remember that $|\Omega|=1$ )

$$
A_{i}=\sqrt{a_{i}}, \quad A_{i, \infty}=\sqrt{a_{i, \infty}}, \quad \overline{A_{i}}=\int_{\Omega} A_{i} d x, \quad i=1,2,3,4 .
$$

Finally, we denote $\|f\|_{2}^{2}=\int_{\Omega} f^{2} d x$ for a given function $f: \Omega \rightarrow \mathbb{R}$.

\section{A-priori estimates}

In this section, we establish a polynomially growing $L^{\infty}$ estimate (proposition 2.1) for the solution of eq. (1.3)-(1.6). We start with the

Lemma 2.1 (A-priori estimates due to the decay of the entropy) Let $a_{i}, i=1,2,3,4$, be solutions of the system (1.3)-(1.6) with initial data such that $a_{i, 0} \ln \left(a_{i, 0}\right) \in L^{1}([0,1])$. Then, for all $T>0$ (and with $M_{i}$ defined in (1.9)),

$$
\begin{aligned}
\left\|\partial_{x} A_{i}\right\|_{L^{2}([0, T] \times[0,1])}^{2} & \leq \frac{1}{4 d_{i}} \int_{0}^{1} \sum_{j=1}^{4} a_{j, 0} \ln \left(a_{j, 0}\right) d x+4 e^{-1}:=C_{4, i}, \\
\sup _{t \in[0, T]}\left\|a_{i} \ln \left(a_{i}\right)\right\|_{L^{1}([0,1])} & \leq \int_{0}^{1} \sum_{i=j}^{4} a_{j, 0} \ln \left(a_{j, 0}\right) d x+5 e^{-1}:=C_{5}, \\
\sup _{t \in[0, T]}\left\|A_{i}\right\|_{L^{2}([0,1])}^{2} & \leq M_{i} .
\end{aligned}
$$

Proof of Lemma 2.1. Integration of the entropy dissipation (1.11) yields

$$
\int_{\Omega} \sum_{i=1}^{4} a_{i} \ln \left(a_{i}\right) d x(T)+4 \sum_{i=1}^{4} d_{i} \int_{0}^{T} \int_{\Omega}\left|\partial_{x} A_{i}\right|^{2} d x d t \leq \int_{\Omega} \sum_{i=1}^{4} a_{i, 0} \ln \left(a_{i, 0}\right) d x
$$

so that (since $-a_{i}\left|\ln \left(a_{i}\right)\right| \leq e^{-1}$ ), estimates (2.1) and (2.2) hold. Then, estimate (2.3) is just the conservation of masses. 
Lemma 2.2 (A-priori bounds in $\left.\boldsymbol{L}^{\mathbf{2}}([\mathbf{0}, \boldsymbol{T}] \times[\mathbf{0}, \mathbf{1}])\right)$ For $i=1,2,3,4$, the solutions $a_{i}$ of $(1.3)-(1.6)$ with initial data $a_{i, 0} \ln \left(a_{i, 0}\right) \in L^{1}([0,1])$ satisfy for $T>0$,

$$
\left\|a_{i}\right\|_{L^{2}([0, T] \times[0,1])}^{2} \leq C_{6, i}(1+T),
$$

where the constants $C_{6, i}$ are stated explicitly in appendix 5.

Proof of Lemma 2.2. Note first that

$$
\left|A_{i}(t, x)-\int_{0}^{1} A_{i}(t, y) d y\right|=\left|\int_{0}^{1} \int_{u=y}^{x} \partial_{u} A_{i}(t, u) d u d y\right| \leq \int_{u=0}^{1}\left|\partial_{u} A_{i}(t, u)\right| d u \text {. }
$$

Hence

$$
\left|A_{i}(t, x)\right|^{2} \leq 2 \int_{0}^{1}\left|\partial_{u} A_{i}(t, u)\right|^{2} d u+2 \int_{0}^{1}\left|A_{i}(t, u)\right|^{2} d u,
$$

which yields (2.4) (using (2.3) and (2.1)), thanks to the following computation:

$$
\begin{aligned}
\left\|a_{i}\right\|_{L^{2}([0, T] \times[0,1])}^{2} & \leq \int_{0}^{T}\left(\sup _{y \in[0,1]}\left|A_{i}(t, y)\right|^{2}\right)\left(\int_{0}^{1}\left|A_{i}(t, x)\right|^{2} d x\right) d t \\
& \leq 2 M_{i} \int_{0}^{T} \int_{0}^{1}\left|\partial_{u} A_{i}(t, u)\right|^{2} d u d t+2 M_{i}^{2} T \leq C_{6, i}(1+T) .
\end{aligned}
$$

The next technical lemma provides classical polynomially growing bounds for the solution of the 1D heat equation, which can be proven in an elementary way.

The main steps of this proof are explained in appendix 5 , together with a formula for the constant $C_{7}$ which appears in (2.6).

Lemma 2.3 (Explicit $L^{r}$ bounds $(r \geq 1)$ for the $1 D$ heat equation) Let a denote the solution of the $1 D$ heat equation $(t>0, x \in[0,1]$, with constant diffusivity $d_{a}$ ) with homogeneous Neumann boundary condition, i.e.

$$
\partial_{t} a-d_{a} \partial_{x x} a=g, \quad \partial_{x} a(t, 0)=\partial_{x} a(t, 1)=0,
$$

and assume for the initial data $a(0, x)=a_{0}(x)$ and for the source term $g(t, x)$ that

$$
a_{0} \in L^{\infty}([0,1]), \quad g \in L^{p}([0,+\infty) \times[0,1]) .
$$

Then, for the exponents $r, p \geq 1$ and $q \in[1,3)$ satisfying $\frac{1}{r}+1=\frac{1}{p}+\frac{1}{q}$ and for all $T>0$, the norm $\|a\|_{L^{r}([0, T] \times[0,1])}$ grows at most polynomially in $T$ like

$$
\|a\|_{L^{r}([0, T] \times[0,1])} \leq T^{1 / r}\left\|a_{0}\right\|_{L^{\infty}[0,1]}+C_{7}\left(1+T^{\frac{1}{q}+\frac{1}{2}}\right)\|g\|_{L^{p}([0, T] \times[0,1])} .
$$


Next, we apply lemma 2.3 to the right-hand side $g=a_{1} a_{3}-a_{2} a_{4}$ of our system, which is bounded in $L^{1}$ by lemma 2.2. As result, we obtain an $L^{r}$ bound with $r<3$ on the $a_{i}$ and thus an improved bound on $g$. Hence, after three iterations (detailed below), we obtain that the $L^{\infty}$ norm increases at most polynomially in time:

Proposition 2.1 Let $a_{i}, i=1,2,3,4$, be solutions of the system (1.3)-(1.6) with bounded initial data $a_{i, 0} \in L^{\infty}(\Omega)$. Then, for $T>0$,

$$
\left\|a_{i}\right\|_{L^{\infty}([0, T] \times[0,1])} \leq C_{8, i}\left(1+T^{\frac{21}{2}}\right) .
$$

The constants $C_{8, i}$ and the constants in the proof are stated in appendix 5.

Proof of Proposition 2.1. By lemma 2.2, we have

$$
\left\|a_{1} a_{3}-a_{2} a_{4}\right\|_{L^{1}([0, T] \times[0,1])} \leq \frac{1}{2} \sum_{i=1}^{4} C_{6, i}(1+T) .
$$

Then, by lemma 2.3 with $p=1$ and $r=q \in[1,3)$, for $i=1,2,3,4$,

$$
\begin{aligned}
\left\|a_{i}\right\|_{L^{r}([0, T] \times[0,1])} & \leq T^{\frac{1}{r}}\left\|a_{i, 0}\right\|_{L^{\infty}[0,1]}+\frac{C_{7}}{2} \sum_{i=1}^{4} C_{6, i}\left(1+T^{\frac{1}{r}+\frac{1}{2}}\right)(1+T), \\
& \leq\left(\left\|a_{i, 0}\right\|_{L^{\infty}[0,1]}+\frac{3}{2} C_{7} \sum_{i=1}^{4} C_{6, i}\right)\left(1+T^{\frac{1}{r}+\frac{3}{2}}\right) .
\end{aligned}
$$

Next, for any $s \in[2,3)$,

$$
\left\|a_{1} a_{3}-a_{2} a_{4}\right\|_{L^{\frac{s}{2}([0, T] \times[0,1])}} \leq C_{15}\left(1+T^{\frac{2}{s}+3}\right) .
$$

Using again lemma 2.3, but with $p=\frac{s}{2}, q \in[1,3)$ and $r \in[1, \infty)$, it follows that

$$
\begin{aligned}
\left\|a_{i}\right\|_{L^{r}([0, T] \times[0,1])} & \leq T^{\frac{1}{r}}\left\|a_{i, 0}\right\|_{L^{\infty}[0,1]}+C_{7} C_{15}\left(1+T^{\frac{1}{q}+\frac{1}{2}}\right)\left(1+T^{\frac{2}{s}+3}\right) \\
& \leq C_{16, i}\left(1+T^{\frac{1}{r}+\frac{9}{2}}\right),
\end{aligned}
$$

since $T^{\frac{1}{q}+\frac{1}{2}} T^{\frac{2}{s}+3}=T^{\frac{1}{r}+\frac{9}{2}}$, and with the constants $C_{16, i}$ given in appendix 5 .

Then, for $s \in[2, \infty)$, we see that

$$
\begin{aligned}
\left\|a_{1} a_{3}-a_{2} a_{4}\right\|_{L^{\frac{s}{2}([0, T] \times[0,1])}} & \leq \frac{1}{2} \sum_{i=1}^{4}\left\|a_{i}\right\|_{L^{s}([0, T] \times[0,1])}^{2} \\
& \leq \sum_{i=1}^{4} C_{16, i}^{2}\left(1+T^{\frac{2}{s}+9}\right)
\end{aligned}
$$


and, secondly, by a last application of lemma 2.3 with $p=\frac{s}{2}, r=\infty$, and $1=\frac{1}{p}+\frac{1}{q}$

$$
\begin{aligned}
\left\|a_{i}\right\|_{L^{\infty}([0, T] \times[0,1])} & \leq\left\|a_{i, 0}\right\|_{L^{\infty}[0,1]}+C_{7} \sum_{i=1}^{4} C_{16, i}^{2}\left(1+T^{\frac{1}{q}+\frac{1}{2}}\right)\left(1+T^{\frac{2}{s}+9}\right) \\
& \leq C_{8, i}\left(1+T^{\frac{21}{2}}\right),
\end{aligned}
$$

since $T^{\frac{1}{q}+\frac{1}{2}} T^{\frac{2}{s}+9}=T^{\frac{21}{2}}$, and with the constant $C_{8, i}$ defined in appendix 5 .

\section{Entropy/entropy dissipation estimate}

In this section, we prove proposition 3.1, which details an entropy/entropydissipation estimate for $E\left(a_{i}\right), D\left(a_{i}\right)$ defined in (1.11). The proof uses the technical (but elementary) lemmata 3.1 and 3.2. Despite being lengthy, we believe that the lemmata 3.1 and 3.2 provide a strategy which extends to more general reaction-diffusion systems. In particular, in the special case of spatial-independent (nonnegative) concentrations, lemma 3.1 establishes a control of a $L^{2}$-distance towards the steady state in terms of a reaction term, which - due to the conservation laws (1.7) - can't cease until the steady state is reached. Lemma 3.2 generalizes this control to spatial-dependent concentrations.

We begin with the :

Lemma 3.1 Let $A_{i, \infty}, i=1,2,3,4$, denote the positive square roots of the steady state (1.10). Let $\overline{A_{i}} \geq 0$ be constants satisfying the conservation laws (1.7), i.e. ${\overline{A_{j}}}^{2}+{\overline{A_{k}}}^{2}=A_{j, \infty}^{2}+A_{k, \infty}^{2}$ for $(j, k) \in(\{1,3\},\{2,4\})$. Then,

$$
\sum_{i=1}^{4}\left\|\overline{A_{i}}-A_{i, \infty}\right\|_{2}^{2} \leq C_{9}\left\|\overline{A_{1}} \overline{A_{3}}-\overline{A_{2}} \overline{A_{4}}\right\|_{2}^{2},
$$

where $C_{9}$ is given in appendix 5 .

Proof of Lemma 3.1. The proof exploits the ansatz

$$
{\overline{A_{i}}}^{2}=A_{i, \infty}^{2}\left(1+\mu_{i}\right)^{2}, \quad-1 \leq \mu_{i}, \quad \text { for } i=1,2,3,4 .
$$

The conservation laws (1.7), more precisely the relations

$$
A_{1, \infty}^{2}\left(2 \mu_{1}+\mu_{1}^{2}\right)+(-1)^{i} A_{i, \infty}^{2}\left(2 \mu_{i}+\mu_{i}^{2}\right)=0, \quad i=2,3,4,
$$

allow to express $\mu_{2}, \mu_{3}$, and $\mu_{4}$ as functions of $\mu_{1}$ :

$$
\mu_{i}=\mu_{i}\left(\mu_{1}\right)=-1+\sqrt{1-(-1)^{i} \frac{A_{1, \infty}^{2}}{A_{i, \infty}^{2}}\left(2 \mu_{1}+\mu_{1}^{2}\right)}, \quad i=2,3,4 .
$$


The function $\mu_{1} \mapsto \mu_{3}\left(\mu_{1}\right)$ is monotone increasing, while $\mu_{1} \mapsto \mu_{2}\left(\mu_{1}\right)$ and $\mu_{1} \mapsto \mu_{4}\left(\mu_{1}\right)$ are monotone decreasing. Moreover, $\mu_{i}\left(\mu_{1}\right)=0$ if and only if $\mu_{1}=0$ (for $\left.i=2,3,4\right)$.

Since $\mu_{2}\left(\mu_{1}\right), \mu_{3}\left(\mu_{1}\right), \mu_{4}\left(\mu_{1}\right)$ are real, $\mu_{1}$ is restricted to

$$
\mu_{1, \min } \leq \mu_{1} \leq \mu_{1, \max }
$$

with

$$
\mu_{1, \min }=-1+\sqrt{1-\frac{\min \left\{A_{1, \infty}^{2}, A_{3, \infty}^{2}\right\}}{A_{1, \infty}^{2}}}, \mu_{1, \max }=-1+\sqrt{1+\frac{\min \left\{A_{2, \infty}^{2}, A_{4, \infty}^{2}\right\}}{A_{1, \infty}^{2}}} .
$$

Due to the above monotonicity properties, we see that

$$
\begin{aligned}
& -1 \leq \mu_{3}\left(\mu_{1, \min }\right) \leq \mu_{3}(0)=0 \leq \mu_{3}\left(\mu_{1, \max }\right), \\
& -1 \leq \mu_{i}\left(\mu_{1, \max }\right) \leq \mu_{i}(0)=0 \leq \mu_{i}\left(\mu_{1, \min }\right), \quad i=2,4 .
\end{aligned}
$$

We now quantify how $\mu_{1} \mapsto \mu_{i}\left(\mu_{1}\right)$ (for $\left.i=2,3,4\right)$ are "close to proportional" to $\mu_{1}$. In particular, for $\mu_{3}$, we Taylor-expand

$$
\sqrt{1+\frac{A_{1, \infty}^{2}}{A_{3, \infty}^{2}}\left(2 \mu_{1}+\mu_{1}^{2}\right)}=1+\frac{1+\zeta}{\sqrt{1+\left(A_{1, \infty}^{2} / A_{3, \infty}^{2}\right)\left(2 \zeta+\zeta^{2}\right)}} \frac{A_{1, \infty}^{2}}{A_{3, \infty}^{2}} \mu_{1},
$$

for some $\zeta \in\left(0, \mu_{1}\right)$, and consider the remainder

$$
R_{3}\left(\mu_{1}\right)=\frac{A_{1, \infty}(1+\zeta)}{\sqrt{1+\left(A_{1, \infty}^{2} / A_{3, \infty}^{2}\right)\left(2 \zeta+\zeta^{2}\right)}}=\frac{A_{3, \infty}^{2}}{A_{1, \infty}} \frac{1}{\mu_{1}}\left(-1+\sqrt{1+\frac{A_{1, \infty}^{2}}{A_{3, \infty}^{2}}\left(2 \mu_{1}+\mu_{1}^{2}\right)}\right),
$$

for $\mu_{1} \in\left[\mu_{1, \min }, \mu_{1, \max }\right]$. It is straightforward that $R_{3}\left(\mu_{1}\right)$ is continuous at $\mu_{1}=0$ with $R_{3}(0)=A_{1, \infty}$, and monotone increasing or decreasing in $\mu_{1} \in\left[\mu_{1, \min }, \mu_{1, \max }\right]$ if and only if $A_{1, \infty}<A_{3, \infty}$ or $A_{1, \infty}>A_{3, \infty}$, respectively. Therefore,

$$
\begin{array}{ll}
0<R_{3}\left(\mu_{1, \min }\right)<R_{3}\left(\mu_{1, \max }\right) \leq A_{3, \infty}, & \text { for } \quad A_{1, \infty} \leq A_{3, \infty}, \\
0<R_{3}\left(\mu_{1, \max }\right)<R_{3}\left(\mu_{1, \min }\right)<2 A_{1, \infty}, & \text { for } \quad A_{1, \infty} \geq A_{3, \infty},
\end{array}
$$

so that

$$
0<R_{3} \leq \max \left\{2 A_{1, \infty}, A_{3, \infty}\right\} .
$$

For $\mu_{2}$ (and analogously for $\mu_{4}$ ), we expand

$$
\sqrt{1-\frac{A_{1, \infty}^{2}}{A_{2, \infty}^{2}}\left(2 \mu_{1}+\mu_{1}^{2}\right)}=1-\frac{1+\zeta}{\sqrt{1-\left(A_{1, \infty}^{2} / A_{2, \infty}^{2}\right)\left(2 \zeta+\zeta^{2}\right)}} \frac{A_{1, \infty}^{2}}{A_{2, \infty}^{2}} \mu_{1},
$$


for some $\zeta \in\left(0, \mu_{1}\right)$, and consider the remainder

$$
R_{2}\left(\mu_{1}\right)=\frac{A_{1, \infty}(1+\zeta)}{\sqrt{1-\left(A_{1, \infty}^{2} / A_{2, \infty}^{2}\right)\left(2 \zeta+\zeta^{2}\right)}}=-\frac{A_{2, \infty}^{2}}{A_{1, \infty}} \frac{1}{\mu_{1}}\left(1-\sqrt{1-\frac{A_{1, \infty}^{2}}{A_{2, \infty}^{2}}\left(2 \mu_{1}+\mu_{1}^{2}\right)}\right),
$$

which is continuous with $R_{2}(0)=A_{1, \infty}$, and increases with respect to $\mu_{1}$. Therefore,

$$
0<R_{2}\left(\mu_{1, \min }\right)<R_{2}\left(\mu_{1, \max }\right) \leq A_{1, \infty}+\sqrt{A_{1, \infty}^{2}+\min \left\{A_{2, \infty}^{2}, A_{4, \infty}^{2}\right\}},
$$

so that finally,

$$
0<R_{2}, R_{4} \leq A_{1, \infty}+\sqrt{A_{1, \infty}^{2}+\min \left\{A_{2, \infty}^{2}, A_{4, \infty}^{2}\right\}} .
$$

Using the ansatz (3.2) to expand (3.1) (and using the identity $A_{1, \infty} A_{3, \infty}=$ $\left.A_{2, \infty} A_{4, \infty}\right)$, we see that in order to prove lemma 3.1 , we only have to establish that

$$
\frac{A_{1, \infty}^{2} \mu_{1}^{2}+A_{2, \infty}^{2} \mu_{2}^{2}+A_{3, \infty}^{2} \mu_{3}^{2}+A_{4, \infty}^{2} \mu_{4}^{2}}{A_{1, \infty}^{2} A_{3, \infty}^{2}\left(\mu_{1}+\mu_{3}+\mu_{1} \mu_{3}-\mu_{2}-\mu_{4}-\mu_{2} \mu_{4}\right)^{2}} \leq C_{9}
$$

for $\mu_{1} \in\left[\mu_{1, \min }, \mu_{1, \max }\right]$.

Considering the numerator of (3.11), we estimate thanks to (3.9), (3.10) that

$$
\sum_{i=1}^{4} A_{i, \infty}^{2} \mu_{i}^{2} \leq \mu_{1}^{2} A_{1, \infty}^{2}\left(1+\frac{R_{2}^{2}}{A_{2, \infty}^{2}}+\frac{R_{3}^{2}}{A_{3, \infty}^{2}}+\frac{R_{4}^{2}}{A_{4, \infty}^{2}}\right) \leq \mu_{1}^{2} A_{1, \infty}^{2} A_{3, \infty}^{2} C_{9},
$$

where $C_{9}$ is given in the appendix 5. Regarding the denominator of (3.11), we assume first that $\mu_{1}<0$. Then, thanks to the properties of monotonicity of $\mu_{1} \mapsto \mu_{i}\left(\mu_{1}\right)$, we observe in the sum $\mu_{1}+\mu_{3}+\mu_{1} \mu_{3}+\left(-\mu_{2}\right)+\left(-\mu_{4}\right)+$ $\left(-\mu_{2} \mu_{4}\right)$ that only the term $\mu_{1} \mu_{3}$ is nonnegative and all the other terms are nonpositive. Moreover, we know in this case that $-1 \leq \mu_{1}$ and $-1 \leq \mu_{3}$; and therefore $\mu_{3} \leq-\mu_{1} \mu_{3}$, implying

$$
\mu_{1}+\mu_{3}+\mu_{1} \mu_{3}-\mu_{2}-\mu_{4}-\mu_{2} \mu_{4} \leq \mu_{1}-\mu_{2}-\mu_{4}-\mu_{2} \mu_{4} \leq-\left|\mu_{1}\right|
$$

If we secondly consider the case $\mu_{1}>0$, only the term $-\mu_{2} \mu_{4}$ is nonpositive and $-1 \leq \mu_{2}$ as well as $-1 \leq \mu_{4}$, therefore $\mu_{2} \leq-\mu_{2} \mu_{4}$ and

$$
\mu_{1}+\mu_{3}+\mu_{1} \mu_{3}-\mu_{2}-\mu_{4}-\mu_{2} \mu_{4} \geq \mu_{1}+\mu_{3}+\mu_{1} \mu_{3}-\mu_{4} \geq\left|\mu_{1}\right| \text {. }
$$

Altogether, by (3.13) and (3.14), we estimate the denominator of (3.11) by

$$
A_{1, \infty}^{2} A_{3, \infty}^{2}\left(\mu_{1}+\mu_{3}+\mu_{1} \mu_{3}-\mu_{2}-\mu_{4}-\mu_{2} \mu_{4}\right)^{2} \geq A_{1, \infty}^{2} A_{3, \infty}^{2} \mu_{1}^{2},
$$

which proves (with (3.12)) that we can take the constant (5.4), and then the lemma 3.1 is obtained. 
The following lemma extends Lemma 3.1 to nonnegative functions $A_{i}$ which satisfy the conservation laws (1.7).

Lemma 3.2 Let $A_{i, \infty}, i=1,2,3,4$, denote the positive square roots of the steady state (1.10), and $A_{i}$ be measurable, nonnegative functions satisfying the conservation laws (1.7), i.e. $\overline{A_{j}^{2}}+\overline{A_{k}^{2}}=M_{j k}=A_{j, \infty}^{2}+A_{k, \infty}^{2}$ for $(j, k) \in$ $(\{1,3\},\{2,4\})$. Then,

$$
\sum_{i=1}^{4}\left\|A_{i}-A_{i, \infty}\right\|_{2}^{2} \leq C_{10}\left\|A_{1} A_{3}-A_{2} A_{4}\right\|_{2}^{2}+C_{11} \sum_{i=1}^{4}\left\|A_{i}-\overline{A_{i}}\right\|_{2}^{2},
$$

where

$$
C_{10}=\max \left\{C_{9}, \max _{j=1,3}\left\{\frac{4 M}{M_{j 2} M_{j 4}}\right\}, \max _{k=2,4}\left\{\frac{4 M}{M_{1 k} M_{3 k}}\right\}\right\},
$$

with $C_{9}$ defined in (5.4) and

$$
C_{11}=\left\{\begin{array}{l}
C_{10}\left(\sqrt{M_{14} M_{32}}+M\right), \text { if } \sqrt{\overline{A_{i}^{2}}} \leq \varepsilon_{i} \text { for some } i=1,2,3,4 \\
C_{9} \sqrt{M_{14} M_{32}}\left(1+\max _{i=1,2,3,4}\left\{\frac{2 \sqrt{M}}{\varepsilon_{i}}\right\}\right)+\max _{i=1,2,3,4}\left\{\frac{2 A_{i, \infty}}{\varepsilon_{i}}\right\}, \text { else. }
\end{array}\right.
$$

Here

$$
\begin{gathered}
\varepsilon_{j}=\frac{\sqrt{M M_{j 2} M_{j 4}}}{M_{j 2}+M_{j 4}}\left(\sqrt{1+\frac{M_{j 2}+M_{j 4}}{2 M}}-1\right), \quad j=1,3, \\
\varepsilon_{k}=\frac{\sqrt{M M_{1 k} M_{3 k}}}{M_{1 k}+M_{3 k}}\left(\sqrt{1+\frac{M_{1 k}+M_{3 k}}{2 M}}-1\right), \quad k=2,4 .
\end{gathered}
$$

Proof of lemma 3.2. In order to apply lemma 3.1, we expand around the mean values

$$
A_{i}=\overline{A_{i}}+\delta_{i}(x), \quad \overline{\delta_{i}}=0, \quad i=1,2,3,4,
$$

and consider the ansatz in lemma 3.1:

$$
\overline{A_{i}^{2}}=A_{i, \infty}^{2}\left(1+\mu_{i}\right)^{2}, \quad-1 \leq \mu_{i},
$$

which preserves the relations (3.3) and thus all the sequel of lemma 3.1.

The ansatz (3.20), (3.21) implies readily for the right-hand side of (3.15) that

$$
\left\|A_{i}-\overline{A_{i}}\right\|_{2}^{2}=\overline{A_{i}^{2}}-{\overline{A_{i}}}^{2}=\overline{\delta_{i}^{2}}
$$


Since

$$
\frac{\overline{\delta_{i}^{2}}}{\sqrt{\overline{A_{i}^{2}}}+\overline{A_{i}}}=\sqrt{\overline{A_{i}^{2}}}-\overline{A_{i}}
$$

it follows that

$$
\overline{A_{i}}=A_{i, \infty}\left(1+\mu_{i}\right)-\frac{1}{\sqrt{\overline{A_{i}^{2}}}+\overline{A_{i}}} \overline{\delta_{i}^{2}} .
$$

For the left-hand side of (3.15), we use (3.24) to expand

$$
\left\|A_{i}-A_{i, \infty}\right\|_{2}^{2}=A_{i, \infty}^{2} \mu_{i}^{2}+\frac{2 A_{i, \infty}}{\sqrt{\overline{A_{i}^{2}}}+\overline{A_{i}}} \overline{\delta_{i}^{2}} .
$$

Thus the expansions in terms of $\overline{\delta_{i}^{2}}$ is unbounded for vanishing $\overline{A_{i}^{2}} \geq{\overline{A_{i}}}^{2}$ and we consider firstly the

- Case $\overline{A_{i}^{2}} \geq \varepsilon_{i}^{2}$ : (leaving the case for small $\overline{A_{i}^{2}}$ for later). We factorize

$$
\begin{aligned}
\left\|A_{1} A_{3}-A_{2} A_{4}\right\|_{2}^{2} & =\left\|\overline{A_{1}} \overline{A_{3}}-\overline{A_{2}} \overline{A_{4}}\right\|_{2}^{2}+2\left(\overline{A_{1}} \overline{A_{3}}-\overline{A_{2}} \overline{A_{4}}\right)\left(\overline{\delta_{1} \delta_{3}}-\overline{\delta_{2} \delta_{4}}\right) \\
& +\left\|\overline{A_{1}} \delta_{3}+\overline{A_{3}} \delta_{1}+\delta_{1} \delta_{3}-\overline{A_{2}} \delta_{4}-\overline{A_{4}} \delta_{2}-\delta_{2} \delta_{4}\right\|_{2}^{2} .
\end{aligned}
$$

Since $\overline{A_{i}} \leq \sqrt{\overline{A_{i}^{2}}}$ by Jensen's inequality and $\overline{A_{1}^{2}} \overline{A_{3}^{2}} \leq M_{14} M_{32}, \overline{A_{2}^{2}} \overline{A_{4}^{2}} \leq$ $M_{14} M_{32}$ by the conservation laws (1.7), we estimate the second term on the right-hand side of (3.26) using Young's inequality:

$$
\begin{aligned}
2\left(\overline{A_{1}} \overline{A_{3}}-\overline{A_{2}} \overline{A_{4}}\right)\left(\overline{\delta_{1} \delta_{3}}-\overline{\delta_{2} \delta_{4}}\right) & \geq-\left|\overline{A_{1}} \overline{A_{3}}-\overline{A_{2}} \overline{A_{4}}\right|\left(\overline{\delta_{1}^{2}}+\overline{\delta_{2}^{2}}+\overline{\delta_{3}^{2}}+\overline{\delta_{4}^{2}}\right) \\
& \geq-\sqrt{M_{14} M_{32}}\left(\overline{\delta_{1}^{2}}+\overline{\delta_{2}^{2}}+\overline{\delta_{3}^{2}}+\overline{\delta_{4}^{2}}\right) .
\end{aligned}
$$

Then, we insert (3.24) (recalling $\left.A_{1, \infty} A_{3, \infty}=A_{2, \infty} A_{4, \infty}\right)$ into

$$
\begin{aligned}
& \left\|\overline{A_{1}} \overline{A_{3}}-\overline{A_{2}} \overline{A_{4}}\right\|_{2}^{2}=A_{1, \infty}^{2} A_{3, \infty}^{2}\left(\mu_{1}+\mu_{3}+\mu_{1} \mu_{3}-\mu_{2}-\mu_{4}-\mu_{2} \mu_{4}\right)^{2} \\
& -2\left(\sqrt{\overline{\overline{A_{1}^{2}}} \overline{\overline{A_{3}^{2}}}}-\sqrt{\overline{\overline{A_{2}^{2}}} \overline{A_{4}^{2}}}\right)\left(\frac{\sqrt{\overline{A_{3}^{2}}} \overline{\delta_{1}^{2}}}{\sqrt{\overline{A_{1}^{2}}}+\overline{A_{1}}}+\frac{\sqrt{\overline{A_{1}^{2}}} \overline{\delta_{3}^{2}}}{\sqrt{\overline{A_{3}^{2}}}+\overline{\overline{A_{3}}}}-\frac{\overline{\delta_{1}^{2}} \overline{\delta_{3}^{2}}}{\left(\sqrt{\overline{A_{1}^{2}}}+\overline{A_{1}}\right)\left(\sqrt{\overline{A_{3}^{2}}}+\overline{A_{3}}\right)}\right. \\
& \left.-\frac{\sqrt{\overline{A_{4}^{2}}} \overline{\delta_{2}^{2}}}{\sqrt{\overline{A_{2}^{2}}}+\overline{A_{2}}}-\frac{\sqrt{\overline{A_{2}^{2}}} \overline{\delta_{4}^{2}}}{\sqrt{\overline{A_{4}^{2}}}+\overline{A_{4}}}+\frac{\overline{\delta_{2}^{2}} \overline{\delta_{4}^{2}}}{\left(\sqrt{\overline{A_{2}^{2}}}+\overline{A_{2}}\right)\left(\sqrt{\overline{A_{4}^{2}}}+\overline{A_{4}}\right)}\right) \\
& +\left\|\frac{\sqrt{\overline{A_{3}^{2}}} \overline{\delta_{1}^{2}}}{\sqrt{\overline{A_{1}^{2}}}+\overline{A_{1}}}+\cdots+\frac{\overline{\delta_{2}^{2}} \overline{\delta_{4}^{2}}}{\left(\sqrt{\overline{A_{2}^{2}}}+\overline{A_{2}}\right)\left(\sqrt{\overline{A_{4}^{2}}}+\overline{A_{4}}\right)}\right\|_{2}^{2} .
\end{aligned}
$$


For the second factor on the right-hand side of (3.28), we estimate like above

$$
\left|\sqrt{\overline{A_{1}^{2}} \overline{A_{3}^{2}}}-\sqrt{\overline{A_{2}^{2}} \overline{A_{4}^{2}}}\right| \leq \sqrt{M_{14} M_{32}}
$$

and use (3.23) to compute

$$
\frac{\sqrt{\overline{A_{3}^{2}}} \overline{\delta_{1}^{2}}}{\sqrt{\overline{A_{1}^{2}}}+\overline{A_{1}}}+\frac{\sqrt{A_{1}^{2}}}{\sqrt{\overline{A_{3}^{2}}}+\overline{A_{3}^{2}}}-\frac{\overline{\delta_{1}^{2}} \overline{\delta_{3}^{2}}}{\left(\sqrt{\overline{A_{1}^{2}}}+\overline{A_{1}}\right)\left(\sqrt{\overline{A_{3}^{2}}}+\overline{A_{3}}\right)}=\frac{1}{2} \frac{\sqrt{A_{3}^{2}}+\overline{A_{3}}}{\sqrt{\overline{A_{1}^{2}}}+\overline{A_{1}}} \frac{1}{\delta_{1}^{2}}+\frac{\sqrt{\overline{A_{1}^{2}}}+\overline{A_{1}}}{\sqrt{\overline{A_{3}^{2}}}+\overline{A_{3}}}
$$

and we compute in the same way the product proportional to $\overline{\delta_{2}^{2} \delta_{4}^{2}}$. Thus, by $\overline{A_{i}} \leq \sqrt{\overline{A_{i}^{2}}}<\sqrt{M}$ for all $i=1,2,3$, we obtain

$$
\begin{gathered}
-\sqrt{M_{14} M_{32}}\left|\frac{\sqrt{\overline{A_{3}^{2}}}+\overline{A_{3}}}{\sqrt{\overline{A_{1}^{2}}}+\overline{A_{1}}} \overline{\delta_{1}^{2}}+\frac{\sqrt{\overline{A_{1}^{2}}}+\overline{A_{1}}}{\sqrt{\overline{A_{3}^{2}}}+\overline{A_{3}}} \overline{\delta_{3}^{2}}-\frac{\sqrt{\overline{A_{4}^{2}}}+\overline{A_{4}}}{\sqrt{\overline{A_{2}^{2}}}+\overline{A_{2}}}-\frac{\sqrt{\overline{A_{2}^{2}}}+\overline{A_{2}}}{\sqrt{\bar{A}_{4}^{2}}+\overline{A_{4}}}\right| \\
\geq-\sqrt{M_{14} M_{32}} \max _{i=1,2,3,4}\left\{\frac{2 \sqrt{M}}{\sqrt{\overline{A_{i}^{2}}}}\right\}\left(\overline{\delta_{1}^{2}}+\overline{\delta_{2}^{2}}+\overline{\delta_{3}^{2}}+\overline{\delta_{4}^{2}}\right) .
\end{gathered}
$$

Therefore, inserting (3.25) into the left-hand side of (3.15) and combining (3.22) and (3.26)-(3.29) for the right-hand side of (3.15) we have to prove that

$$
\begin{aligned}
& \sum_{i=1}^{4} A_{i, \infty}^{2} \mu_{i}^{2} \leq C_{10} A_{1, \infty}^{2} A_{3, \infty}^{2}\left(\mu_{1}+\mu_{3}+\mu_{1} \mu_{3}-\mu_{2}-\mu_{4}-\mu_{2} \mu_{4}\right)^{2} \\
+ & \left(C_{11}-C_{10} \sqrt{M_{14} M_{32}}\left(1+\max _{i}\left\{\frac{2 \sqrt{M}}{\sqrt{\overline{A_{i}^{2}}}}\right\}\right)-\max _{i}\left\{\frac{2 A_{i, \infty}}{\sqrt{\overline{A_{i}^{2}}}+\overline{A_{i}}}\right\}\right) \sum_{i=1}^{4} \overline{\delta_{i}^{2}} .
\end{aligned}
$$

When $C_{10} \geq C_{9}$ with $C_{9}$ stated in (5.4), then Lemma 3.1 (see (3.11)) implies

$$
\sum_{i=1}^{4} A_{i, \infty}^{2} \mu_{i}^{2} \leq C_{9} A_{1, \infty}^{2} A_{3, \infty}^{2}\left(\mu_{1}+\mu_{3}+\mu_{1} \mu_{3}-\mu_{2}-\mu_{4}-\mu_{2} \mu_{4}\right)^{2}
$$

and we look for

$$
C_{11} \geq C_{9} \sqrt{M_{14} M_{32}}\left(1+\max _{i=1,2,3,4}\left\{\frac{2 \sqrt{M}}{\sqrt{A_{i}^{2}}}\right\}\right)+\max _{i=1,2,3,4}\left\{\frac{2 A_{i, \infty}}{\sqrt{A_{i}^{2}}}\right\} .
$$

We now treat the case

- Case $\overline{A_{i}^{2}} \leq \varepsilon_{i}^{2}$ : More precisely, we suppose that $\overline{A_{i}^{2}} \leq \varepsilon_{i}^{2}$, where $\varepsilon_{i}$ are constants to be specified later. In particular for $\overline{A_{1}} \leq{\overline{A_{1}^{2}}}^{1 / 2} \leq \varepsilon_{1}$, we estimate (using $\overline{A_{3}}<\sqrt{M}, \overline{A_{2}} \leq \sqrt{M_{12}}, \overline{A_{4}} \leq \sqrt{M_{14}}$ and ${\overline{A_{2}}}^{2}{\overline{A_{4}}}^{2}=\left(\overline{A_{2}^{2}}-\right.$ $\left.\overline{\delta_{2}^{2}}\right)\left(\overline{A_{4}^{2}}-\overline{\delta_{4}^{2}}\right)$ with $(1.7)$ for the product $\left.\overline{A_{2}^{2}} \overline{A_{4}^{2}}\right)$ that

$$
\begin{aligned}
& \left\|\overline{A_{1}} \overline{A_{3}}-\overline{A_{2}} \overline{A_{4}}\right\|_{2}^{2}={\overline{A_{1}}}^{2}{\overline{A_{3}}}^{2}-2 \overline{A_{1}} \overline{A_{3}} \overline{A_{2}} \overline{A_{4}}+{\overline{A_{2}}}^{2}{\overline{A_{4}}}^{2} \\
& \geq-2 \varepsilon_{1} \sqrt{M M_{12} M_{14}}+\left(M_{12}-\varepsilon_{1}^{2}\right)\left(M_{14}-\varepsilon_{1}^{2}\right)-\overline{A_{4}^{2}} \overline{\delta_{2}^{2}}-{\overline{A_{2}}}^{2} \overline{\delta_{4}^{2}} .
\end{aligned}
$$


Moreover by (1.7), a straightforward expansion yields

$$
\sum_{i=1}^{4}\left\|A_{i}-A_{i, \infty}\right\|_{2}^{2} \leq 2 M
$$

Thus, combining the left-hand side of (3.15) with (3.32) and the right-

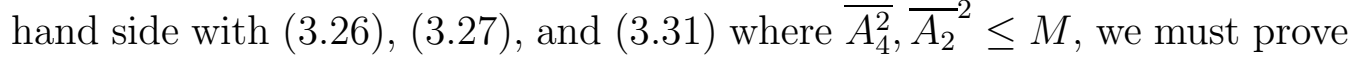
that

$$
\begin{aligned}
2 M \leq & C_{10}\left(M_{12} M_{14}-2 \varepsilon_{1} \sqrt{M M_{12} M_{14}}-\varepsilon_{1}^{2}\left(M_{12}+M_{14}\right)+\varepsilon_{1}^{4}\right) \\
& +\left(C_{11}-C_{10} \sqrt{M_{14} M_{32}}-C_{10} M\right)\left(\overline{\delta_{1}^{2}}+\overline{\delta_{2}^{2}}+\overline{\delta_{3}^{2}}+\overline{\delta_{4}^{2}}\right) .
\end{aligned}
$$

We treat the first bracket on the right-hand side of (3.33). After neglecting $\varepsilon_{1}^{4}$, we denote the nonnegative solution of the (in terms of $\varepsilon$ ) quadratic equation $x y-2 \varepsilon \sqrt{M x y}-\varepsilon^{2}(x+y)=h$ for $0 \leq h \leq x y$ by

$$
\varepsilon(x, y, h):=-\frac{\sqrt{M x y}}{x+y}+\sqrt{\frac{M x y}{(x+y)^{2}}+\frac{x y-h}{x+y}} .
$$

In the present case, where $x=M_{12}$ and $y=M_{14}$, choosing in particular $h=\frac{x y}{2}$ confirms (3.33) with

$$
\left.\begin{array}{l}
C_{10} \geq \frac{2 M}{h}=\frac{4 M}{M_{12} M_{14}} \\
C_{11} \geq C_{10}\left(\sqrt{M_{14} M_{32}}+M\right)
\end{array}\right\} \text { for } \sqrt{\overline{A_{1}^{2}}} \leq \varepsilon_{1}:=\varepsilon\left(M_{12}, M_{14}, \frac{M_{12} M_{14}}{2}\right) .
$$

Similarly, for the cases $\overline{A_{i}^{2}} \leq \varepsilon_{i}^{2}, i=2,3,4$, we obtain the same $C_{11}$ and

$$
\begin{array}{ll}
C_{10} \geq \frac{4 M}{M_{32} M_{34}}, & \text { for } \sqrt{\overline{A_{3}^{2}}} \leq \varepsilon_{3}:=\varepsilon\left(M_{32}, M_{34}, \frac{M_{32} M_{34}}{2}\right), \\
C_{10} \geq \frac{4 M}{M_{1 k} M_{3 k}}, & \text { for } \sqrt{\overline{A_{k}^{2}}} \leq \varepsilon_{k}:=\varepsilon\left(M_{1 k}, M_{3 k}, \frac{M_{1 k} M_{3 k}}{2}\right), \quad k=2,4,
\end{array}
$$

and this yields (3.16) and (3.17).

We are now in position to state the entropy/entropy-dissipation estimate for $E, D$ defined in (1.11), which holds for admissible functions regardless if or if not they are solutions (at a given time $t$ ) of eq. (1.3) - (1.6).

Proposition 3.1 Let $a_{i}$ be (measurable) functions from $[0,1]$ to $\mathbb{R}$ such that $0 \leq a_{i} \leq\left\|a_{i}\right\|_{L^{\infty}([0,1])}$, and $\int_{0}^{1}\left(a_{j}+a_{k}\right)=M_{j k}$ for $(j, k) \in(\{1,3\},\{2,4\})$. Then,

$$
D\left(a_{i}\right) \geq \frac{4}{C_{12}} \min \left\{\frac{1}{C_{10}}, \frac{\min \left\{d_{1}, d_{2}, d_{3}, d_{4}\right\}}{C_{11} P([0,1])}\right\}\left(E\left(a_{i}\right)-E\left(a_{i, \infty}\right)\right)
$$


where $P([0,1])$ is the Poincaré constant of interval $[0,1], C_{10} \equiv C_{10}\left(M_{j k}\right)$ is defined in (3.16), $C_{11} \equiv C_{11}\left(M_{j k}\right)$ in (3.17), and

$$
C_{12}\left(\left\|a_{i}\right\|_{L^{\infty}([0,1])}, M_{j k}\right)=\max _{i}\left\{\Phi\left(\left\|a_{i}\right\|_{L^{\infty}([0,1])}, a_{i, \infty}\right)\right\} .
$$

Here, $\Phi$ is the function defined by the formula

$$
\Phi(x, y)=\frac{x(\ln (x)-\ln (y))-(x-y)}{(\sqrt{x}-\sqrt{y})^{2}}, \quad \Phi(x, y)=O(\ln (x)) .
$$

Proof of Proposition 3.1. Using the inequality $\left(a_{1} a_{3}-a_{2} a_{4}\right)\left(\ln \left(a_{1} a_{3}\right)-\right.$ $\left.\ln \left(a_{2} a_{4}\right)\right) \geq 4\left(A_{1} A_{3}-A_{2} A_{4}\right)^{2}$ and Poincaré's inequality, we obtain the estimate

$$
D\left(a_{i}\right) \geq 4\left\|A_{1} A_{3}-A_{2} A_{4}\right\|_{2}^{2}+\sum_{i=1}^{4} \frac{4 d_{i}}{P(\Omega)}\left\|A_{i}-\overline{A_{i}}\right\|_{2}^{2} .
$$

We show in the sequel that the right-hand side of (3.40) is bounded below by the relative entropy $E\left(a_{i}\right)-E\left(a_{i, \infty}\right)$.

First, we use the conservation laws (1.7) to rewrite the relative entropy as

$$
E\left(a_{i}\right)-E\left(a_{i, \infty}\right)=\int_{\Omega} \sum_{i=1}^{4}\left(a_{i} \ln \frac{a_{i}}{a_{i, \infty}}-\left(a_{i}-a_{i, \infty}\right)\right) d x
$$

and we use the boundedness of the function $\Phi$ defined in (3.39), (see $[8$, lemma 2.1]) to estimate

$$
E\left(a_{i}\right)-E\left(a_{i, \infty}\right) \leq C_{12} \sum_{i=1}^{4}\left\|A_{i}-A_{1, \infty}\right\|_{2}^{2},
$$

with $C_{12}$ as defined in (3.38). The statement of proposition 3.1 follows now from lemma 3.2 by comparison with (3.40).

\section{Estimates of convergence towards equilibrium}

In this section, we use the estimates of the two previous sections in order to obtain proposition 4.2 and theorem 1.1. We begin with a Cziszar-Kullback type inequality relating convergence in entropy with $L^{1}$ convergence.

Proposition 4.1 For all (measurable) functions $a_{i}:[0,1] \rightarrow \mathbb{R}^{+}, i=1,2,3,4$, for which $\int_{0}^{1}\left(a_{j}+a_{k}\right)=M_{j k}$ for $(j, k) \in(\{1,3\},\{2,4\})$, we have the inequality

$$
2 \sqrt{2}\left(E\left(a_{i}\right)-E\left(a_{i, \infty}\right)\right) \geq \sum_{i=1}^{4} M_{i}^{-1}\left\|a_{i}-a_{i, \infty}\right\|_{1}^{2},
$$

with $M_{i}$ defined in (1.9) and for the entropy functional $E\left(a_{i}\right)$ defined in (1.11). 
Proof of Proposition 4.1. We define

$$
q\left(a_{i}\right)=a_{i} \ln a_{i}-a_{i}
$$

and rewrite

$$
E\left(a_{i}\right)-E\left(a_{i, \infty}\right)=\sum_{i=1}^{4} \int_{\Omega} a_{i} \ln \frac{a_{i}}{\overline{a_{i}}} d x+\sum_{i=1}^{4}\left(q\left(a_{i}\right)-q\left(a_{i, \infty}\right)\right) .
$$

Using the conservation laws (1.7), we define moreover

$$
Q_{j k}\left(M_{j k}, \overline{a_{j}}\right)=q\left(\overline{a_{j}}\right)+q\left(M_{j k}-\overline{a_{j}}\right)=Q_{j k}\left(M_{j k}, \overline{a_{k}}\right) \text { for } \overline{a_{j}}, \overline{a_{k}} \in\left[0, M_{j k}\right],
$$

and rewrite the second sum on the right-hand side of (4.1) as

$$
\begin{aligned}
& \sum_{i=1}^{4}\left(q\left(a_{i}\right)-q\left(a_{i, \infty}\right)\right)= \\
& \quad=Q_{j k}\left(M_{j k}, \overline{a_{j}}\right)-Q_{j k}\left(M_{j k}, a_{j, \infty}\right)+Q_{j^{\prime} k^{\prime}}\left(M_{j^{\prime} k^{\prime}}, \overline{a_{j^{\prime}}}\right)-Q_{j^{\prime} k^{\prime}}\left(M_{j^{\prime} k^{\prime}}, a_{j^{\prime}, \infty}\right) \\
& \quad=Q_{j k}\left(M_{j k}, \overline{a_{k}}\right)-Q_{j k}\left(M_{j k}, a_{k, \infty}\right)+Q_{j^{\prime} k^{\prime}}\left(M_{j^{\prime} k^{\prime}}, \overline{a_{k^{\prime}}}\right)-Q_{j^{\prime} k^{\prime}}\left(M_{j^{\prime} k^{\prime}}, a_{k^{\prime}, \infty}\right),
\end{aligned}
$$

with $j \neq j^{\prime}$ and $j, j^{\prime} \in\{1,3\}$ and $k \neq k^{\prime}$ and $k, k^{\prime} \in\{2,4\}$. Since the derivatives $Q_{j k}^{\prime}$ and $Q_{j k}^{\prime \prime}$ satisfy

$$
Q_{j k}^{\prime}\left(M_{j k}, \overline{a_{j}}\right)+Q_{j^{\prime} k^{\prime}}^{\prime}\left(M_{j^{\prime} k^{\prime}}, \overline{a_{j^{\prime}}}\right)=Q_{j k}^{\prime}\left(M_{j k}, \overline{a_{k}}\right)+Q_{j^{\prime} k^{\prime}}^{\prime}\left(M_{j^{\prime} k^{\prime}}, \overline{a_{k^{\prime}}}\right)=0,
$$

and

$$
Q_{j k}^{\prime \prime}\left(M_{j k}, \overline{a_{j}}\right) \geq \frac{4}{M_{j k}}, \quad Q_{j k}^{\prime \prime}\left(M_{j k}, \overline{a_{k}}\right) \geq \frac{4}{M_{j k}},
$$

we Taylor-expand (4.1) (where the first order terms vanish due to $\overline{a_{1}}-a_{1, \infty}=$ $\overline{a_{3}}-a_{3, \infty}$ and $\overline{a_{2}}-a_{2, \infty}=\overline{a_{4}}-a_{4, \infty}$, respectively) and get

$$
\sum_{i=1}^{4}\left(q\left(a_{i}\right)-q\left(a_{i, \infty}\right)\right) \geq \sum_{i=1}^{4} M_{i}^{-1}\left|a_{i}-a_{i, \infty}\right|^{2} .
$$

Secondly, for the first term on the right-hand side of (4.1), we estimate with the classical Cziszar-Kullback-Pinsker inequality (Cf. [5])

$$
\int_{\Omega} a_{i} \ln \frac{a_{i}}{\overline{a_{i}}} d x \geq \frac{1}{2 \overline{a_{i}}}\left\|a_{i}-\overline{a_{i}}\right\|_{1}^{2}
$$

for which moreover $\overline{a_{i}} \leq M_{i}$. Alltogether, we obtain (by Young's inequality $\left.\left\|a_{i}-a_{i, \infty}\right\|_{1}^{2} \leq \sqrt{2}\left\|a_{i}-\overline{a_{i}}\right\|_{1}^{2}+2 \sqrt{2}\left|\overline{a_{i}}-a_{i, \infty}\right|^{2}\right)$

$$
E\left(a_{i}\right)-E\left(a_{i, \infty}\right) \geq \sum_{i=1}^{4} \frac{\left\|a_{i}-a_{i, \infty}\right\|_{1}^{2}}{2 \sqrt{2} M_{i}} .
$$

This ends the proof of proposition 4.1 . 
We now are in a position to state the

Proposition 4.2 Let $d_{i}>0$ for $i=1,2,3,4$ be strictly positive diffusion rates. Let the initial data $a_{i, 0}$ be nonnegative functions of $L^{\infty}([0,1])$ with strictly positive masses $M_{j k}$ for $(j, k) \in(\{1,3\},\{2,4\})$. Then, the unique classical solution $(t, x) \mapsto a_{i}(t, x)$ to eq. (1.3) - (1.6) satisfies (for $M_{i}$ defined in (1.9) and $E$ in (1.11)) the decay (1.12), i.e.

$$
\sum_{i=1}^{4} M_{i}^{-1}\left\|a_{i}(t, \cdot)-a_{i, \infty}\right\|_{L^{1}([0,1])}^{2} \leq 2 \sqrt{2}\left(E\left(a_{i, 0}\right)-E\left(a_{i, \infty}\right)\right) e^{-\frac{C_{1} t}{\ln (e+t)}}
$$

with a constant $C_{1}$ which can be computed explicitly (Cf. appendix 5).

Proof of Proposition 4.2. Thanks to the entropy identity $\frac{d}{d t} E\left(a_{i}\right)=$ $-D\left(a_{i}\right)$, Proposition 3.1 yields

$$
\frac{d}{d t} \ln \left(E\left(a_{i}\right)-E\left(a_{i, \infty}\right)\right) \geq \frac{4}{C_{12}(t)} \min \left\{\frac{1}{C_{10}}, \frac{\min \left\{d_{1}, d_{2}, d_{3}, d_{4}\right\}}{C_{11} P}\right\},
$$

where $C_{12}(t)=\max _{i=1,2,3,4}\left\{\Phi\left(\left\|a_{i}\right\|_{L^{\infty}([0, t] \times[0,1])}, a_{i, \infty}\right)\right\}$ with $\Phi(x, y)$ defined in (3.39) (this function is monotone increasing in $x$, Cf. [8], lemma 2.1), and $C_{10}, C_{11}$ and $P$ defined in proposition 3.1.

Moreover, it is easy to see that for $k>1$,

$$
\Phi(k y, y)=\frac{k \ln (k)-(k-1)}{(\sqrt{k}-1)^{2}} \leq \frac{\sqrt{k}+1}{\sqrt{k}-1} \ln (k), \quad \forall k>1 .
$$

Note that the factor $(\sqrt{k}+1) /(\sqrt{k}-1)$ is strictly monotone decreasing in $k$.

Next, we know thanks to lemma 2.1 that $\left\|a_{i}\right\|_{L^{\infty}([0, t] \times[0,1])} \leq C_{8, i}\left(1+t^{\frac{21}{2}}\right)$. Thus, in order to apply (4.3) with e.g. $k \geq 2$, we estimate $\left\|a_{i}\right\|_{L^{\infty}([0, t] \times[0,1])} \leq$ $\max \left\{C_{8, i}, 2 a_{i, \infty}\right\}\left(1+t^{\frac{21}{2}}\right)$ so that

$$
\begin{gathered}
\Phi\left(\left\|a_{i}\right\|_{L^{\infty}([0, t] \times[0,1])}, a_{i, \infty}\right) \leq \Phi\left(\max \left\{\frac{C_{8, i}}{a_{i, \infty}}, 2\right\}\left(1+t^{\frac{21}{2}}\right) a_{i, \infty}, a_{i, \infty}\right) \\
\leq \frac{\sqrt{2}+1}{\sqrt{2}-1}\left(\ln \left(\max \left\{\frac{C_{8, i}}{a_{i, \infty}}, 2\right\}\right)+\ln \left(1+t^{\frac{21}{2}}\right)\right)
\end{gathered}
$$

and therefore

$$
C_{12}(t) \leq(\sqrt{2}+1)^{2}\left(\max _{i=1,2,3,4}\left\{\ln \left(\frac{C_{8, i}}{a_{i, \infty}}\right), \ln 2\right\}+\ln \left(1+t^{\frac{21}{2}}\right)\right) .
$$

Next, we notice that

$$
\int_{0}^{T} \frac{d t}{\max \left\{\ln \frac{C_{8, i}}{a_{i, \infty}}, \ln 2\right\}+\ln \left(1+t^{\frac{21}{2}}\right)} \geq \frac{1}{\left(\max \left\{\frac{C_{8, i}}{a_{i, \infty}}, \ln 2\right\}+\frac{21}{2}\right)} \frac{T}{\ln (e+T)},
$$


since both sides vanish at $T=0$ and the time-derivatives of the left-hand side can be estimated below by

$$
\begin{aligned}
& \geq \frac{1}{\max \left\{\ln \frac{C_{8, i}}{a_{i, \infty}}, \ln 2\right\}+\frac{21}{2} \ln (e+T)} \geq \frac{1}{\left(\max \left\{\frac{C_{8, i}}{a_{i, \infty}}, \ln 2\right\}+\frac{21}{2}\right)} \frac{1}{\ln (e+T)} \\
& >\frac{1}{\left(\max \left\{\frac{C_{8, i}}{a_{i, \infty}}, \ln 2\right\}+\frac{21}{2}\right)} \frac{1}{\ln (e+T)}\left(1-\frac{T}{e+T} \frac{1}{\ln (e+T)}\right)
\end{aligned}
$$

which is the time-derivative of the right-hand side of (4.4).

Finally, estimate (1.12) follows from integrating (4.2) on $[0, T]$ and the Cziszar-Kullback type proposition 4.1.

We now present the proof of Theorem 1.1, which is based on interpolation properties, and a second application of the entropy/entropy-dissipation estimate (Proposition 3.1).

Proof of Theorem 1.1. To establish an $H^{1}$ bound on the solution of equations (1.3)-(1.6), Proposition 2.1 and (2.1) yield

$$
T \inf _{t \in[0, T]}\left\|\partial_{x} a_{i}\right\|_{L^{2}([0,1])}^{2} \leq\left\|\partial_{x} a_{i}\right\|_{L^{2}([0, T] \times[0,1])}^{2} \leq 4 C_{4, i} C_{8, i}\left(1+T^{\frac{21}{2}}\right),
$$

for all $T>0$. Since the function $\left(T^{-1}+T^{\frac{19}{2}}\right)$ assumes its minimum value at time $T=(2 / 19)^{2 / 21}$, there exists a time $\tau \in\left[0,(2 / 19)^{2 / 21}\right]$ when

$$
\left\|\partial_{x} a_{i}(\tau)\right\|_{L^{2}([0,1])}^{2} \leq 4 C_{4, i} C_{8, i} \frac{21}{2}\left(\frac{2}{19}\right)^{\frac{19}{21}} .
$$

Next, multiplying eq. (1.3) formally with $\partial_{x x} a_{i}$ yields with Young's inequality

$\frac{d}{d t} \int_{0}^{1}\left|\partial_{x} a_{i}\right|^{2} d x+d_{i} \int_{0}^{1}\left(\partial_{x x} a_{i}\right)^{2} d x \leq \frac{1}{4 d_{i}}\left\|a_{1} a_{3}-a_{2} a_{4}\right\|_{L^{2}([0,1])}^{2}+d_{i} \int_{0}^{1}\left(\partial_{x x} a_{i}\right)^{2} d x$.

We integrate over a time interval $T>(2 / 19)^{2 / 21} \geq \tau$ this formula and obtain

$$
\left\|\partial_{x} a_{i}(T)\right\|_{L^{2}([0,1])}^{2} \leq\left\|\partial_{x} a_{i}(\tau)\right\|_{L^{2}([0,1])}^{2}+\frac{1}{4 d_{i}}\left\|a_{1} a_{3}-a_{2} a_{4}\right\|_{L^{2}([0, T] \times[0,1])}^{2} .
$$

Using the bound (2.9), i.e. $\left\|a_{1} a_{3}-a_{2} a_{4}\right\|_{L^{2}([0, T] \times[0,1])} \leq \sum_{i=1}^{4} C_{16, i}^{2}\left(1+T^{\frac{19}{2}}\right)$, we obtain

$$
\left\|\partial_{x} a_{i}(T)\right\|_{L^{2}(\Omega)}^{2}<C_{17}\left(1+T^{19}\right) \quad \text { for } T>(2 / 19)^{2 / 21},
$$

with the constant $C_{17}$ given in the appendix 5. This formal argument can be made rigorous by approximations of the solution (see e.g. [24]). 
Next, we use (see e.g. [32]) the Gagliardo-Nirenberg-Moser interpolation inequality

$$
\left\|a_{i}\right\|_{L^{\infty}([0,1])} \leq G([0,1])\left\|\partial_{x} a_{i}\right\|_{L^{2}([0,1])}^{\frac{1}{2}}\left\|a_{i}\right\|_{L^{2}([0,1])}^{\frac{1}{2}} .
$$

Then, interpolating the almost exponentially decaying $L^{1}$ norm of Proposition 4.2 for $T>(2 / 19)^{2 / 21} \geq \tau$, we get

$$
\begin{aligned}
& \left\|a_{i}(T)\right\|_{L^{\infty}([0,1])} \leq a_{i, \infty}+\left\|a_{i}-a_{i, \infty}\right\|_{L^{\infty}([0,1])} \\
& \leq a_{i, \infty}+G([0,1])\left\|\partial_{x}\left(a_{i}-a_{i, \infty}\right)\right\|_{L^{2}([0,1])}^{\frac{1}{2}}\left\|a_{i}-a_{i, \infty}\right\|_{L^{\infty}([0,1])}^{\frac{1}{4}}\left\|a_{i}-a_{i, \infty}\right\|_{L^{1}([0,1])}^{\frac{1}{4}} \\
& \leq C_{13, i},
\end{aligned}
$$

where (4.5), Proposition 2.1 and Proposition 4.2 lead to the constants $C_{13, i}$ given by (5.5) in appendix 5. Moreover, for $0<\tau \leq(2 / 19)^{2 / 21}$, the $L^{\infty}$ bound of Theorem 1.1, i.e. the value of $C_{2, i}$ (5.1) follows from Proposition 2.1. Finally, using this global $L^{\infty}$ bound, the right-hand side of (4.2) is bounded below by a constant and the exponential decay stated in the theorem can be obtained by the standard Gronwall's lemma.

\section{Appendix}

In order to convince the reader that all constants in this work are explictly computable, we provide the following formulas:

\section{Lemma 5.1 (Explicit constants)}

$$
\begin{aligned}
& C_{1}= \frac{4 \min \left\{\frac{1}{C_{10}}, \frac{\min \left\{d_{1}, d_{2}, d_{3}, d_{4}\right\}}{C_{11} P([0,1])}\right\}}{(\sqrt{2}+1)^{2}\left(\max _{i=1,2,3,4}\left\{\frac{C_{8, i}}{a_{i, \infty}}, \ln 2\right\}+\frac{21}{2}\right)}, \\
& C_{2, i} \leq\left\{\begin{array}{c}
\frac{21}{19} C_{8, i}, 0<t \leq\left(\frac{2}{19}\right)^{2 / 21} \\
C_{13, i}, t>\left(\frac{2}{19}\right)^{2 / 21}
\end{array}\right. \\
& C_{3}=\left(\begin{array}{c}
4 \min \left\{\frac{1}{C_{10}}, \frac{\min _{i=1,2,3,4}\left\{d_{i}\right\}}{C_{11} P([0,1])}\right\} \\
\max _{i=1,2,3,4}\left\{\Phi\left(C_{13, i}, a_{i, \infty}\right\}\right)
\end{array}\right) \\
& C_{6, i}=2 M_{i}\left(M_{i}+C_{4, i}\right), \\
& C_{7}=d_{a}^{-\frac{1}{q}} 2^{\frac{4 q^{2}-3 q-2}{q(2 q-1)}\left(\frac{\pi}{q}\right)^{\frac{2+q}{2 q(2 q-1)}}\left(\frac{1}{3-q}\right)^{\frac{2+q}{q(2 q-1)}}\left(\frac{1}{2+q}\right)^{\frac{q-3}{q(2 q-1)}}} \\
&+ d_{a}^{\frac{1}{2}} 2^{3+\frac{2}{q}}\left(\frac{1}{2+q}\right)^{\frac{1}{q}},
\end{aligned}
$$


(5.3)

$$
C_{8, i}=\left\|a_{i, 0}\right\|_{L^{\infty}[0,1]}+3 C_{7} \sum_{i=1}^{4} C_{16, i}^{2}
$$

(5.4) $C_{9}=\frac{1+\max \left\{4 \frac{A_{1, \infty}^{2}}{A_{3, \infty}^{2}}, 1\right\}}{A_{3, \infty}^{2}}+\left(\frac{1}{A_{2, \infty}^{2}}+\frac{1}{A_{4, \infty}^{2}}\right) \frac{\left(A_{1, \infty}+\sqrt{A_{1, \infty}+\min \left\{A_{2, \infty}^{2}, A_{4, \infty}^{2}\right.}\right)^{2}}{A_{3, \infty}^{2}}$,

$$
\begin{aligned}
& C_{13, i}=a_{i, \infty}+G(\Omega) C_{17}^{\frac{1}{4}} C_{8, i}^{\frac{1}{4}}\left(2^{\frac{3}{2}} M_{i}\left(E\left(a_{i, 0}\right)-E\left(a_{i, \infty}\right)\right)\right)^{\frac{1}{8}} 3^{\frac{1}{4}} C_{14}, \\
& C_{14}=\sup _{t \in[0, \infty)}\left\{\left(1+t^{\frac{59}{2}}\right)^{\frac{1}{4}} \exp \left(-\frac{t}{\ln (e+t)} \frac{\min \left\{\frac{1}{C_{10}}, \frac{\min \left\{d_{1}, d_{2}, d_{3}, d_{4}\right\}}{C_{11} P([0,1])}\right\}}{2(\sqrt{2}+1)^{2}\left(\max _{i=1,2,3,4}\left\{\frac{C_{8, i}}{a_{i, \infty}}\right\}+\frac{21}{2}\right)}\right)\right\}
\end{aligned}
$$

$$
C_{15}=\sum_{i=1}^{4}\left(\left\|a_{i, 0}\right\|_{L^{\infty}[0,1]}+\frac{3}{2} C_{7} \sum_{i=1}^{4} C_{6, i}\right)^{2}
$$

$$
C_{16, i}=\left\|a_{i, 0}\right\|_{L^{\infty}[0,1]}+3 C_{7} C_{15}
$$

$$
C_{17}=4 C_{4, i} C_{8, i} \frac{21}{2}\left(\frac{2}{19}\right)^{\frac{19}{21}}+\frac{1}{2 d_{i}}\left(\sum_{i=1}^{4} C_{16, i}^{2}\right)^{2}
$$

where $M_{i}$ is defined in (1.9), $C_{4, i}$ is given in (2.1), $C_{10}$ is defined in (3.16) (depending on $C_{9}$ given in (5.4)), $C_{11}$ is defined in (3.17), $C_{15}$ in (5.6), $C_{16, i}$ in (5.7), and the function $\Phi$ is given in (3.39). Moreover, $P([0,1])$ denotes the Poincaré constant of $[0,1]$ and $G([0,1])$ denotes the Gagliardo-NirenbergMoser constant in (4.6).

We also provide a short proof of Lemma 2.3 for the sake of completeness.

Proof of Lemma 2.3. The proof uses Fourier series, which simplify when (2.5) is mirrored evenly around $x=0$, i.e. when the functions are extended like

$$
\tilde{a}(t, x)= \begin{cases}a(t, x) & x \in[0,1] \\ a(t,-x) & x \in[-1,0]\end{cases}
$$

and when $\tilde{g}$ and $\tilde{a_{0}}$ are defined analogously. Then, the eigenvalue-problem $\tilde{\varphi}_{x x}=\lambda \tilde{\varphi}$ on $[-1,1]$ with homogeneous Neumann boundary and periodicity conditions is satisfied by the eigenvalue-eigenfunction pairs

$$
\left(\lambda_{k}, \tilde{\varphi}_{k}(x)\right)=\left(-(k \pi)^{2}, \cos (k \pi x)\right) \quad \text { for } \quad k=0,1,2, \ldots
$$


and yields the Fourier representation

$$
\begin{aligned}
& \tilde{a}(t, x)=\int_{-1}^{1} \tilde{a_{0}}(y) d y+2 \sum_{k=1}^{\infty} e^{\lambda_{k} d_{a} t}\left(\int_{-1}^{1} \tilde{a_{0}}(y) \tilde{\varphi}_{k}(y) d y\right) \tilde{\varphi}_{k}(x) \\
& \quad+\int_{0}^{t} \int_{-1}^{1} \tilde{g}(s, y) d y d s+2 \sum_{k=1}^{\infty} \int_{0}^{t} e^{\lambda_{k} d_{a}(t-s)}\left(\int_{-1}^{1} \tilde{g}(s, y) \tilde{\varphi}_{k}(y) d y\right) d s \tilde{\varphi}_{k}(x) .
\end{aligned}
$$

Thanks to Poisson's summation formula, we can write down

$$
\begin{aligned}
\tilde{a}(t, x)= & \frac{1}{2 \sqrt{\pi}} \int_{-1}^{1} \tilde{a_{0}}(y) \sum_{k=-\infty}^{\infty} \frac{1}{\sqrt{d_{a} t}} e^{-\frac{(2 k+x-y)^{2}}{4 d_{a} t}} d y \\
& +\frac{1}{2 \sqrt{\pi}} \int_{0}^{t} \int_{-1}^{1} \tilde{g}(s, y) \sum_{k=-\infty}^{\infty} \frac{1}{\sqrt{d_{a}(t-s)}} e^{-\frac{(2 k+x-y)^{2}}{4 d_{a}(t-s)}} d y d s .
\end{aligned}
$$

This yields the estimate

$$
\begin{aligned}
\|\tilde{a}\|_{L^{r}([0, T] \times[-1,1])} \leq & \frac{1}{2 \sqrt{\pi}}\left\|\tilde{a_{0}} *_{x} S\right\|_{L^{r}([0, T] \times[-1,1])} \\
& +\frac{1}{2 \sqrt{\pi}}\left\|\tilde{g} *_{t, x} S\right\|_{L^{r}([0, T] \times[-1,1])},
\end{aligned}
$$

where $S(t, x):=\sum_{k=-\infty}^{\infty} \frac{1}{\sqrt{d_{a} t}} e^{-\frac{(2 k+x)^{2}}{4 d_{a} t}}$ satisfies (for $q \in[1,3)$ )

$$
\|S(t, \cdot)\|_{L^{1}([-1,1])}=2 \sqrt{\pi}, \quad\|S\|_{L^{q}([0, T] \times[-1,1])} \leq C_{7}\left(1+T^{\frac{1}{q}+\frac{1}{2}}\right) .
$$

The second formula of (5.13) can be obtained by using (when $n \neq 0$ )

$$
(2 n+x)^{2} \geq|2 n+x| \geq 2 n-1
$$

in order to estimate

$$
\begin{aligned}
& \|S\|_{L^{q}([0, T] \times[-1,1])} \leq\left\|\left(d_{a} t\right)^{-\frac{1}{2}}\left(e^{-\frac{x^{2}}{4 d_{a} t}}+2 \sum_{n=1}^{\infty} e^{-\frac{2 n-1}{4 d_{a} t}}\right)\right\|_{L^{q}([0, T] \times[-1,1])} \\
& \leq\left\|\left(d_{a} t\right)^{-\frac{1}{2}} e^{-\frac{x^{2}}{4 d_{a} t}}\right\|_{L^{q}([0, T] \times[-1,1])}+2\left\|\left(d_{a} t\right)^{-\frac{1}{2}}\left(e^{1 / 4 d_{a} t}-e^{-1 / 4 d_{a} t}\right)^{-1}\right\|_{L^{q}([0, T] \times[-1,1])} \\
& \leq\left(\int_{0}^{T}\left(d_{a} t\right)^{-q / 2} 2 \sqrt{\pi} \sqrt{\frac{d_{a} t}{q}} \operatorname{erf}\left(\frac{\sqrt{q}}{2 \sqrt{d_{a} t}}\right) d t\right)^{1 / q}+4\left(\int_{0}^{T}\left|2\left(d_{a} t\right)^{1 / 2}\right|^{q} d t\right)^{1 / q} .
\end{aligned}
$$

Returning to (5.12), we can estimate each term in the right-hand side in order to obtain Lemma 2.3, the fourth term being the most difficult. In order to treat it, we apply Young's inequality $\|\tilde{g} * S\|_{L^{r}} \leq\|\tilde{g}\|_{L^{p}}\|S\|_{L^{q}}$ for $\frac{1}{r}+1=\frac{1}{p}+\frac{1}{q}$ and estimate (5.13) for $\|S\|_{L^{q}}$ 


\section{References}

[1] Amann, H.: Global existence for semilinear parabolic systems. J. Reine Angew. Math. 360 (1985), 47-83.

[2] Cáceres, M., Carrillo, J., Toscani, G.: Long-time behavior for a nonlinear fourth order parabolic equation. Trans. Amer. Math. Soc. 357 (2005), no. 3, 1161-1175.

[3] Cáceres, M., Carrillo, J., Goudon, T.: Equilibration rate for the linear inhomogeneous relaxation-time Boltzmann equation for charged particles. Comm. Partial Differential Equations 28 (2003), no. 5-6, 969-989.

[4] Conway, E., Hoff, D., Smoller, J.: Large time behaviour of solutions of systems of nonlinear reaction-diffusion equations. SIAM J. Appl. Math. 35 (1978), no. 1, 1-16.

[5] Csiszár, I.: Eine informationstheoretische Ungleichung und ihre Anwendung auf den Beweis von Markoffschen Ketten. Magyar Tud. Akad. Mat. Kutató Int. Közl 8 (1963), 85-108.

[6] Carrillo, J., Vazquez, J. L.: Fine asymptotics for fast diffusion equations. Comm. Partial Differential Equations 28 (2003), no. 5-6, 1023-1056.

[7] Del Pino, M., Dolbeault, J.: Best constants for Gagliardo-Nirenberg inequalities and applications to nonlinear diffusions. J. Math. Pures Appl. 81 (2002), no. 9, 847-875.

[8] Desvillettes, L., Fellner, K.: Exponential Decay toward Equilibrium via Entropy Methods for Reaction-Diffusion Equations. J. Math. Anal. Appl. 319 (2006), no. 1, 157-176.

[9] Desvillettes, L., Fellner, K., Pierre, M., Vovelle, J.: About Global Existence for Quadratic Systems of Reaction-Diffusion. Adv. Nonlinear Stud. 7 (2007), no. 3, 491-511.

[10] Desvillettes, L., Mouhot, C.: Large time Behavior of the a priori bounds for the solutions to the spatially homogeneous Boltzmann equations with soft potentials. Asymptot. Anal. 54 (2007), no. 3-4, 235-245.

[11] Desvillettes, L., Villani, C.: On the spatially homogeneous Landau equation for hard potentials. II. $H$-theorem and applications. Comm. Partial Differential Equations 25 (2000), no. 1-2, 261-298.

[12] Desvillettes, L., Villani, C.: On the trend to global equilibrium in spatially inhomogeneous entropy-dissipating systems: the linear FokkerPlanck equation. Comm. Pure Appl. Math. 54 (2001), no. 1, 1-42.

[13] Desvillettes, L., Villani, C.: On the trend to global equilibrium for spatially inhomogeneous kinetic systems: the Boltzmann equation. Invent. Math. 159 (2005), no. 2, 245-316.

[14] Fellner, K., Neumann, L., Schmeiser, C.: Convergence to global equilibrium for spatially inhomogeneous kinetic models of non-micro-reversible processes. Monatsh. Math. 141 (2004), no. 4, 289-299. 
[15] Fitzgibbon, W.E., Morgan, J., Sanders, R.: Global existence and boundedness for a class of inhomogeneous semilinear parabolic systems. Nonlinear Anal. 19 (1992), no. 9, 885-899.

[16] Fitzgibbon, W., Hollis, S., Morgan, J.: Stability and Lyapunov functions for reaction-diffusion systems. SIAM J. Math. Anal. 28 (1997), no. 3, 595-610.

[17] Glitzky, A., Gröger, K., Hünlich, R.: Free energy and dissipation rate for reaction-diffusion processes of electrically charged species. Appl. Anal. 60 (1996), no. 3-4, 201-217.

[18] Glitzky, A., HüNlich, R.: Energetic estimates and asymptotics for electro-reaction-diffusion systems. Z. Angew. Math. Mech. 77 (1997), no. $11,823-832$.

[19] GröGER, K.: Free energy estimates and asymptotic behaviour of reactiondiffusion processes. Preprint 20, Institut für Angewandte Analysis und Stochastik, Berlin, 1992.

[20] Haraux, A., Youkana, A.: On a result of K. Masuda concerning reaction-diffusion equations. Tôhoku Math. J. (2) 40 (1988), no. 1, 159-163.

[21] Hollis, S., Martin, R., Pierre, M.: Global existence and boundedness in reaction-diffusion systems. SIAM J. Math. Appl. 18 (1987), 744-761.

[22] Hoshino, H., Yamada, Y.: Asymptotic behavior of global solutions for some reaction-diffusion systems. Nonlinear Anal. 23 (1994), no. 5, 639-650.

[23] Masuda, K.: On the global existence and asymptotic behavior of solution of reaction-diffusion equations. Hokkaido Math. J. 12 (1983), 360-370.

[24] Martin, R. H., Pierre, M.: Nonlinear Reaction-Diffusion Systems. In Nonlinear Equations in Applied Sciences, 363-398. Math. Sci. Engrg. 185. Academic Press, Boston, MA, 1992.

[25] Morgan, J.: Global existence for semilinear parabolic systems. SIAM J. Math. Anal. 20 (1989), no. 5, 1128-1144.

[26] Mulone, G.: Nonlinear stability in fluid-dynamics in the presence of stabilizing effects and the choice of a measure of perturbations. In WASCOM 2003, 12th Conference on Waves and Stability in Continuous Media, 352365. World Sci. Publ., River Edge, NJ, 2004.

[27] Kanel, Y. I., Kirane, M.: Global solutions of Reaction-Diffusion Systems with a Balance Law and Nonlinearities of Exponential Growth. J. Differential Equations 165 (2000), no. 1, 24-41.

[28] Pierre, M.: Weak solutions and supersolutions in $L^{1}$ for reaction-diffusion systems. J. Evol. Eq. 3 (2003), no. 1, 153-168.

[29] Pierre, M., Schmitt, D.: Blowup in reaction-diffusion systems with dissipation of mass. SIAM Rev. 42 (2000), no. 1, 93-106 (electronic).

[30] Rionero, S.: On the stability of binary reaction-diffusion systems. Nuovo Cimento Soc. Ital. Fis. B 119 (2004), no. 7-9, 773-784. 
[31] Rothe, F.: Global Solutions of Reaction-Diffusion Systems. Lecture Notes in Mathematics 1072. Springer-Verlag, Berlin, 1984.

[32] Taylor, M. E.: Partial Differential Equation III - Nonlinear Equations. Applied Mathematical Sciences 117. Springer-Verlag, New York, 1997.

[33] Toscani, G., Villani, C.: Sharp entropy dissipation bounds and explicit rate of trend to equilibrium for the spatially homogeneous Boltzmann equation. Comm. Math. Phys. 203 (1999), no. 3, 667-706.

[34] Toscani, G., Villani, C.: On the trend to equilibrium for some dissipative systems with slowly increasing a priori bounds. J. Statist. Phys. 98 (2000), no. 5-6, 1279-1309.

[35] Villani, C.: Cercignani's conjecture is sometimes true and always almost true. Comm. Math. Phys. 234 (2003), no. 3, 455-490.

[36] WebB, G.F.: Theory of nonlinear age-dependent population dynamics. Monographs and Textbooks in Pure and Applied Mathematics 89. Marcel Dekker, Inc., New York, 1985.

[37] Zelenyak, T.: Stabilization of solutions of boundary value problems for second-order parabolic equations in one space variable. Differentsial'nye Uravneniya 4 (1968), 34-45.

Recibido: 4 de abril de 2006

Revisado: 10 de abril de 2007

Laurent Desvillettes

CMLA - ENS de Cachan

61 Av. du Pdt. Wilson

94235 Cachan Cedex, France

Laurent.Desvillettes@cmla.ens-cachan.fr

Klemens Fellner

DAMTP, CMS University of Cambridge

Wilberforce Road, Cambridge CB3 0WA, UK

on leave of absence from

Faculty of Mathematics

University of Vienna

Nordbergstr. 15, 1090 Wien, Austria

Klemens.Fellner@univie.ac.at

This work has been supported by the European IHP network "HYKE-HYperbolic and Kinetic Equations: Asymptotics, Numerics, Analysis", Contract Number: HPRN-CT2002-00282. K.F. has also been supported by the Austrian Science Fund FWF project P16174-N05, by the Wittgenstein Award 2000 of Peter A. Markowich, and by the KAUST investigator award 2008 of Peter A. Markowich. 